\title{
DETERMINANTES DE FIDELIDAD EN EL SERVICIO TURÍSTICO UN MODELO DE RELACIONES CAUSALES
}

\author{
SILVINA ETCHEVERRÍA \\ ANA MARÍA LEGATO \\ PATRICIA LAURA RÁBAGO
}

FACULTAD DE CIENCIAS ECONÓMICAS, UNICEN - CEA

ARGENTINA. 


\begin{tabular}{c}
\hline SILVINA ETCHEVERRÍA - ANA MARÍA LEGATO - PATRICIA LAURA RÁBAGO \\
$>106$ DETERMINANTES DE FIDELIDAD EN EL SERVICIO TURÍSTICO UN MODELO DE RELACIONES CAUSALES \\
Pags. $104-127$
\end{tabular}

Recibido: 06/05/2014

Aceptado: 10/10/2014

\title{
RESUMEN
}

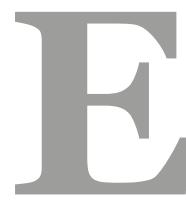

$\mathrm{n}$ el presente trabajo se analizan los factores que determinan la fidelidad del turista que visita la ciudad de Tandil (Argentina) a partir de un modelo de relaciones causales, específicamente, Modelo de Ecuaciones Estructurales. A efectos de la estimación y evaluación del modelo se utilizó una encuesta estructurada a los turistas que visitaron la ciudad, a lo largo de un año calendario. Los resultados obtenidos muestran que la fidelidad del turista aparece como consecuencia tanto de su satisfacción como del potencial del destino percibido por el mismo. Mientras que la satisfacción depende tres constructos: cobertura de necesidades vivenciales, entendidas como el cúmulo de sensaciones que vive el turista producto de la visita y con las cuales finalmente asociará su viaje; cobertura de necesidades funcionales son aquellas ligadas a la logística del viaje y cobertura de necesidades simbólicas, en especial los aspectos vinculados al cuidado del medio ambiente.

Palabras clave: Fidelidad, Turismo, Satisfacción, Ecuaciones Estructurales.

\begin{abstract}
In this study the factors that determine the satisfaction and loyalty of tourists visiting the city of Tandil (Argentina) from a causal model, specifically, Structural Equation Model relations are analyzed. For the purpose of assessment and evaluation model was used a structured survey, to the tourists who visited the city, over a calendar year. The results show that tourist satisfaction depends primarily on the extent to which cover is given to their existential needs, this is a cluster of sensations experienced by the tourist at the instance of the visit and which eventually associate the trip. A second factor is the degree of coverage of functional needs, those are related to the logistics of the trip. Third, symbolic needs play an important role particularly those related to environmental protection aspects. Finally tourist loyalty appears as a result of both satisfaction and perceived potential destination for tourists.
\end{abstract}

Keywords: Satisfaction, Loyalty, Holidays, Structural Equations.

\section{INTRODUCCIÓN}

El turismo es uno de los sectores de actividad de gran importancia desde el punto de vista económico, social y cultural, intensificándose su participación en las economías regionales y 
constituyéndose en motor de desarrollo y crecimiento de aquellos lugares en los que se ejerce este tipo de actividades. En los últimos años ha aumentado considerablemente el movimiento de turistas y la demanda se ha ampliado y diversificado. Gracias a esa diversificación de la demanda, numerosas regiones encuentran en sus recursos naturales, culturales o paisajísticos una base a partir de la cual generar actividad económica, riqueza y desarrollo.

A nivel mundial el turismo ha crecido de manera significativa en los últimos 50 años y, de acuerdo a datos de la Organización Mundial de Turismo, esta "industria sin humo" en pleno crecimiento, experimentó un incremento del 408\% en el período 1960-1980, del 112\% en el período 1980-2000 y un aumento del 151\% entre 2000 y 2012.

En la captación de visitantes, unos destinos compiten fuertemente con otros, de manera que la importancia de sus recursos y la calidad de los mismos no siempre aseguran el éxito futuro.

El presente estudio se llevó a cabo en Tandil, ciudad serrana situada en el sureste de la provincia de Buenos Aires en Argentina. Se caracteriza por poseer un gran número de paseos naturales que son su principal atractivo turístico. Pero también cuenta con una especial oferta relacionada con actividades litúrgicas que incrementan las visitas en fechas cercanas a la Semana Santa y tradicionalmente, ha sido identificada con este tipo de turismo, antes de convertirse en un destino con actividad constante a lo largo del año. En esta ciudad el turismo ha experimentado un crecimiento significativo convirtiéndose en una muy importante actividad local. Cabe consignar, como ejemplo, que las plazas turísticas crecieron un 185 por ciento entre 1999 y $2012^{1}$. A raíz de este crecimiento se aunaron esfuerzos en la organización de esta actividad económica, tanto desde sectores privados como desde el ámbito oficial.

El objetivo de este trabajo es elaborar un modelo cuantitativo, de relaciones causales, que permita poner de manifiesto las variables e indicadores que determinan la fidelización del turista que visita el destino, como también cuantificar o establecer en qué medida influyen dichas variables.

\section{MARCO CONCEPTUAL E HIPÓTESIS.}

En la presente investigación se pretende, con el modelo propuesto, explicar la fidelidad a partir de dos variables fundamentales: satisfacción del cliente y potencial de destino.

\subsection{DESTINO TURÍSTICO Y PRODUCTO INTEGRAL TURÍSTICO}

La actividad turística no puede ser efectivamente abordada si no se tiene en cuenta el

\footnotetext{
${ }^{1}$ Según datos de la Dirección de Turismo del municipio de la ciudad.
} 
carácter multidimensional de la misma, dado que abarca un conjunto complejo de factores heterogéneos que se combinan e interrelacionan. Por lo tanto, deben tenerse en cuenta ciertos elementos que hacen que pueda desarrollarse un destino turístico, entendiéndose por tal, a un espacio geográfico determinado, en el cual se puede identificar un núcleo central que es el elemento principal de las actividades turísticas, además de una serie de bienes y servicios, recursos y equipamientos necesarios para la prestación del servicio.

En tal sentido, el espacio geográfico motivo de la investigación, cuenta con factores básicos del producto turístico dados por el atractivo del lugar, y las actividades que se puedan desarrollar tales como instalaciones en los paseos, facilidades de acceso, seguridad en las prestaciones, cuidado y limpieza de la ciudad, trato recibido del personal del contacto y del ciudadano, y otros elementos que posibilitan el consumo de los servicios como la infraestructura, entretenimientos, comercios, alojamientos y servicios gastronómicos. Oyarzún \& Szmulewicz (2002, p.5) definen el producto integral turístico como el conjunto de todos aquellos elementos, productos y servicios ofertados simultánea e integradamente que son capaces de generar un desplazamiento con fines turísticos.

Considerando que los productos turísticos dependen siempre de la participación de más de un sector, la composición del mismo debe considerar diversos aspectos fundamentales.

En primer lugar los servicios ofrecidos deben reflejar e interpretar las motivaciones del turista, quien busca con su viaje satisfacer tres grandes grupos de necesidades Oyarzún \&. Szmulewicz (2002, p. 6):

- Las necesidades básicas originadas por el viaje que incluye transporte hasta el destino, traslados dentro del mismo, alojamiento y alimentación. A este conjunto se lo denomina necesidades funcionales.

- Las necesidades vinculadas a valores adicionales que ofrece el producto y que tienen un significado psicológico que va más allá de los elementos físicos. Se incluyen en esta categoría aspectos religiosos, históricos y de cuidado medioambientales. A este grupo las denominan necesidades simbólicas.

- Las necesidades asociadas al cúmulo de sensaciones que vivirá el turista producto de la visita y con las cuales finalmente asociará su viaje. Se incluyen en este grupo las percepciones estéticas, el trato recibido por el personal de contacto y del ciudadano y, la seguridad percibida. Éstas se denominan necesidades vivenciales.

\subsection{SATISFACCIÓN Y FIDELIDAD APLICADAS AL TURISMO}

La satisfacción de los clientes es un objetivo fundamental a tener en cuenta en el éxito de cualquier tipo de actividad, pues alude al cumplimiento del deseo o del gusto de quienes experimentan una acción determinada, y esto también es aplicable a un sector como el turístico, en donde la competencia aumenta día a día. Clientes satisfechos son los motores del crecimiento 
del número de visitantes que experimenta un destino turístico, más aún si se considera la importancia que ha adquirido la comunicación en la actualidad donde abundan las tecnologías que facilitan la información prácticamente al instante.

No todos los atributos y dimensiones de los productos poseen la misma importancia a la hora de explicar la satisfacción del cliente. En tal sentido, en trabajos realizados por Kozak \& Rimmington (2000), surgen evidencias respecto de la existencia de atributos o factores del mismo que resultan críticos para explicar la satisfacción. Asimismo, la literatura ha constatado la presencia de determinados elementos de la oferta turística (como el trato recibido o el tipo de relaciones interpersonales implicados en la prestación del servicio) que son independientes de la motivación del viaje y que tienen un gran peso en la valoración final de la experiencia (Bitner et al., 1996; Gundersen \& Olsson, 1996).

Alcanzar la satisfacción del cliente se convierte en una cuestión prioritaria para el destino y para las empresas que allí operan, dado que de ello dependerá que el cliente repita su visita y realice una comunicación positiva del destino a otros posibles clientes (Devesa \& Palacios, 2005).

La satisfacción respecto de un destino turístico, depende tanto de las expectativas que tenga el consumidor-visitante como de la valoración que haga del producto-destino una vez que lo haya consumido. Es, en definitiva, un concepto complejo que resulta de la agregación de numerosas variables que afectan tanto al consumidor, como al propio producto o servicio. Entre las variables que afectan al consumidor es posible mencionar factores personales, culturales, económicos, motivacionales, experiencias previas o actitudes, en tanto que entre las variables que afectan específicamente al producto deberían considerarse las características, atributos, calidad y costos.

Esta complejidad es especialmente relevante en el caso del turismo ya que es necesario considerar tanto elementos tangibles como intangibles (Nowak \& Washburn, 1998). Entre los primeros se destacan los recursos físicos, las infraestructuras hoteleras y de gastronomía, los recursos complementarios, los accesos, las comunicaciones. Entre los elementos intangibles se pueden señalar el trato recibido, la calidad del servicio, las emociones sentidas ante los recursos turísticos visitados, la imagen del lugar o la interacción con otros turistas. Son elementos más difíciles de analizar y de describir pero imprescindibles cuando el objeto bajo análisis consiste en la prestación de servicios, convirtiéndose en determinantes de la satisfacción con mayor importancia que los primeros.

El análisis de la satisfacción evalúa la discrepancia entre el servicio esperado y el experimentado, efectuado siempre como un juicio posterior al disfrute de los servicios. Por lo tanto, para que los turistas se encuentren satisfechos debe considerarse no solo el concepto de necesidad, pues ésta expresa solo la carencia de algunos satisfactores básicos. También se deben tener en cuenta los deseos que motivan la experiencia en el destino, dado que son aquellos satisfactores específicos para dar cumplimiento a las necesidades profundas (Kotler, 1993). 
Por su parte, Spreng, Mackenzie \& Olshavsky, (1996, p. 16): sostienen que los deseos juegan siempre un rol importante en la formación de la satisfacción, definiendo a la misma, como una comparación efectuada por el consumidor entre sus percepciones del desempeño de un producto o servicio con sus expectativas. Asimismo, sostienen que cuando se satisfacen las expectativas de los clientes, y las percepciones y las expectativas son iguales, califican el servicio como satisfactorio. Cuando no se satisfacen las expectativas de los clientes, es decir, si sus expectativas están por encima del servicio que realmente se les ofreció, la calificación será negativa y el cliente calificará al servicio como no satisfactorio. Si el servicio sobrepasa las expectativas de los clientes, la satisfacción será positiva y el cliente calificará su servicio como superior. La satisfacción depende entonces de la brecha entre las expectativas previas del cliente y la valoración que el mismo realice con posterioridad a la utilización del producto o servicio.

Es importante remarcar que tanto las percepciones como las expectativas son subjetivas y, por lo tanto, entran en juego muchas variables de tipo psicológico, pues se trata de procesos en los que coexisten elementos afectivos y cognitivos (Wirtz et al. (2000); Szymansky \& Henard, 2001; Zins, 2002 y Bowen \& Clarke 2002), a los que deben añadirse otros elementos tales como las necesidades y los deseos.

De acuerdo a lo expresado precedentemente, la satisfacción es un estado psicológico ligado a la emoción, que surge de la confirmación o no de las expectativas, unida a los sentimientos previos acerca de la experiencia de consumo. En esta definición resaltan dos componentes: uno cognitivo y otro afectivo (Oliver, 1980).

En lo que se refiere al componente cognitivo de la satisfacción, son fundamentales las percepciones sobre la calidad del servicio y la percepción del individuo sobre si se ha confirmado o no el estándar o los estándares de comparación. Tradicionalmente, se ha utilizado en el estudio de la satisfacción como estándar de comparación el nivel esperado, es decir, el que tiene que ver con la predicción que el cliente hace sobre lo que va a encontrarse. Como consecuencia de la evaluación de los elementos citados (componente cognitivo), surgirían las emociones de los clientes (componente afectivo). Pueden resultar emociones positivas o negativas, que influirán en la satisfacción del cliente respecto al servicio. En tal sentido, Rico (1993, p. 213) define a la satisfacción como un estado de ánimo que crea agrado y complacencia por la realización completa de las necesidades y expectativas creadas. El estado de ánimo de los clientes surge como producto de relacionar las necesidades y expectativas versus el valor y los rendimientos percibidos.

Teniendo en cuenta entonces que para medir la satisfacción en la prestación de servicios se deben analizar una serie de atributos o soportes en los cuales se "tangibiliza" la prestación del servicio, se enuncian las siguientes hipótesis:

H1a: La percepción experimentada en la cobertura de necesidades básicas originadas por el viaje (alojamiento, alimentación, transporte) influye positivamente en la satisfacción. 
H1b: La percepción experimentada en la cobertura de necesidades vinculadas a valores adicionales que tienen un significado psicológico (aspectos religiosos, históricos y de cuidados medioambientales) influye positivamente en la satisfacción.

H1c: La percepción de la cobertura de necesidades vinculadas a las sensaciones que vivirá el turista producto de la visita y con las cuales finalmente asociará su viaje influye positivamente en la satisfacción.

De todos modos, el análisis del proceso de adquisición del servicio no debe analizarse sólo bajo estas hipótesis, sino que se hace necesario evaluar el comportamiento posterior a la compra ya que, tras ésta, el consumidor se sentirá satisfecho o insatisfecho, lo que indudablemente incidirá en su actitud hacia el producto y en sus decisiones futuras (Kotler et al., 1997).

En función de ese sentimiento final, es que el consumidor tomará algunas decisiones importantes como: volver a comprar o no el servicio, comprar o no otros servicios de la misma empresa y/o recomendar o no a sus conocidos el bien o servicio, en este caso particular, aplicado al destino turístico.

Esta última fase tiene también una importancia fundamental en lo que al sector turístico se refiere, como también en el mundo de los viajes. Si el visitante obtiene una sensación de satisfacción, aumenta la posibilidad de repetición de la visita y, por tanto, de fidelidad al destino turístico; puede asimismo, volver a ese lugar para cubrir otra necesidad; y además, influirá de forma decisiva en las futuras decisiones de su entorno social. Por el contrario, si el visitante se va insatisfecho, tal vez se produzca una disonancia que reducirá la probabilidad de repetición y generará una imagen menos positiva del destino entre sus conocidos.

Finalmente, un aspecto que no se debe ignorar al estudiar la posibilidad de que un turista regrese a un destino, es el potencial del mismo. Aspecto que involucra la sensación en el visitante de la factibilidad de realizar otras actividades en una próxima visita o de reiterar las ya realizadas sin que resulte monótono. Un turista que regresa a su lugar de origen con el convencimiento de haber agotado la oferta de actividades que el destino ofrece, probablemente no regresará aunque se sienta satisfecho.

Por todo lo expuesto, se proponen las siguientes hipótesis:

H2a: La satisfacción influye positivamente en la fidelidad del turista.

H2b: El potencial del destino influye positivamente en la fidelidad del turista. 
La Figura 1 resume la vinculación conceptual de las hipótesis planteadas.

Figura 1. Modelo teórico del efecto de las coberturas de necesidades sobre la Satisfacción y la Fidelidad

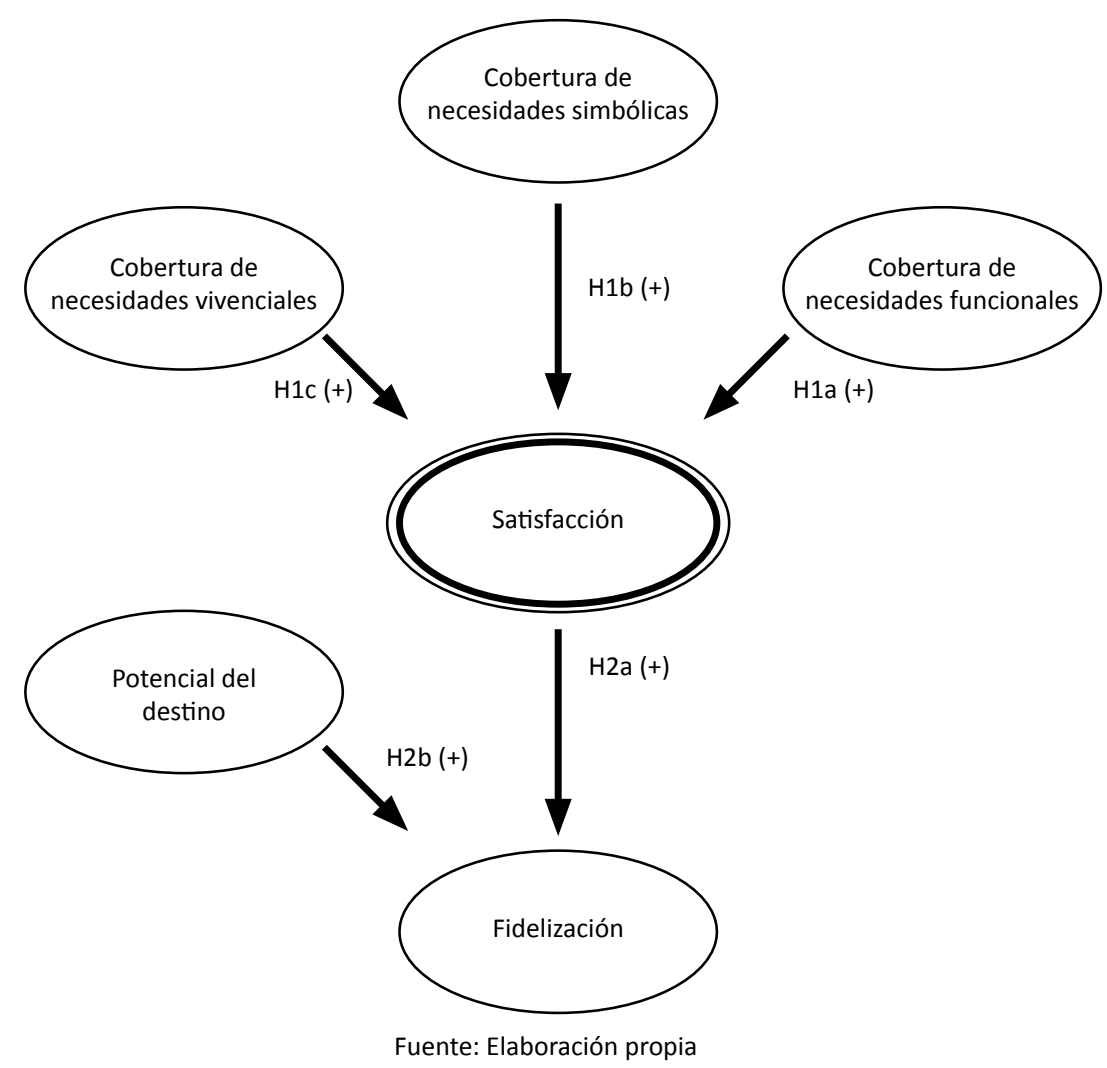

\section{METODOLOGÍA}

\subsection{DISEÑO DE LA INVESTIGACIÓN}

Para responder a los objetivos de la investigación y contrastar las hipótesis planteadas, se desarrolló un estudio empírico cuantitativo de naturaleza causal (Golderberg \& Duncan, 1973), aplicando un cuestionario estructurado en las encuestas llevadas a cabo para tal fin. Las relaciones planteadas en el modelo teórico se estiman mediante el empleo de Modelo de Ecuaciones Estructurales (Structural Equations Modeling, SEM) (Joreskög \& Sörbom, 1979).

La información procede de una encuesta personal realizada a turistas, entendiendo por tal las personas no residentes que han pernoctado al menos una vez en la ciudad. El proceso de recolección de datos se llevó a cabo a lo largo de todo un año, octubre 2011 a octubre 2012, 
a efectos de incluir el período de vacaciones estivales, fiestas religiosa, fines de semanas largos y períodos de menor movimiento turístico, con el fin de minimizar el sesgo producto de la duración de las estadías y del efecto estacional. Modalidad de obtención de datos, que hace a la representatividad de la muestra.

Una vez culminado el trabajo de campo el tamaño de muestra resulta de 203 casos, con un nivel de confianza del 95\%, que proporciona un error muestral de 6,8 \%. La conformación de la muestra exhibe igual proporción de hombres que de mujeres, con edad media de 45,3 años, dispersión de 12,15 años, cifras que muestran una variabilidad de 26,82\%.

\subsection{DEFINICIÓN DE LAS VARIABLES}

En el proceso de diseñar el modelo de trabajo se consideraron dos ideas muy ligadas a la imagen de la ciudad turística. Por un lado el concepto de la ciudad como lugar vinculado a actividades litúrgicas y religiosas, debido a la existencia del Monte Calvario, considerado como el tercero en importancia a nivel mundial. Por otro lado, la percepción de una ciudad inmersa en un paisaje de características singulares, muy conectada al medio ambiente y con posibilidades de desarrollar turismo en la naturaleza. Aspectos estos, que dieron lugar a la definición de los indicadores de la variable Cobertura de necesidades simbólicas.

Una vez completo el relevamiento de los datos se evaluó la relevancia relativa de las preguntas utilizadas para medir cada indicador. Es así como quedó en evidencia que solamente un 1,08\% de los turistas había participado de alguna actividad religiosa. Debido al escaso peso relativo sobre el total de la muestra se decidió obviar la participación de la variable "Valoración de la propuesta religiosa de la ciudad", pues solamente generaba ruido entre la totalidad de las variables consideradas para el modelo.

Finalmente, las variables consideradas a efectos de la investigación, se exhiben en la Tabla 1, conjuntamente con la notación simbólica correspondiente. Las mismas se clasificaron como variables latentes o no observables y variables observables o indicadores. Los indicadores, se midieron utilizando una escala de 1 a 10 puntos. 
Tabla 1. Variables e indicadores considerados en el modelo

\begin{tabular}{|c|c|c|c|c|}
\hline $\begin{array}{l}\text { Variable } \\
\text { latente }\end{array}$ & $\begin{array}{c}\text { Variables } \\
\text { observables }\end{array}$ & Notación & Descriptor & Medida \\
\hline \multirow{5}{*}{$\begin{array}{c}\text { Cobertura de } \\
\text { necesidades } \\
\text { funcionales } \\
\left(\xi_{1}\right)\end{array}$} & NA & $\mathrm{x}_{1}$ & $\begin{array}{l}\text { Valoración de la calidad percibida de } \\
\text { los servicios de alojamiento. }\end{array}$ & Escala de 10 puntos \\
\hline & ACC & $\mathrm{x}_{2}$ & $\begin{array}{l}\text { Valoración de las formas de } \\
\text { acceso a Tandil. }\end{array}$ & Escala de 10 puntos \\
\hline & SG & $x_{3}$ & $\begin{array}{l}\text { Valoración de la calidad percibida } \\
\text { de los servicios gastronómicos. }\end{array}$ & Escala de 10 puntos \\
\hline & $\mathrm{Pr}$ & $x_{4}$ & Valoración del nivel de precios. & Escala de 10 puntos \\
\hline & Ins. Pas & $x_{5}$ & $\begin{array}{l}\text { Valoración de las instalaciones } \\
\text { en los paseos. }\end{array}$ & Escala de 10 puntos \\
\hline \multirow{2}{*}{$\begin{array}{c}\text { Cobertura de } \\
\text { necesidades } \\
\text { simbólicas } \\
\left(\xi_{2}\right) \\
\end{array}$} & MA & $\overline{x_{6}}$ & $\begin{array}{l}\text { Valoración del cuidado del medio } \\
\text { ambiente y los recursos naturales. }\end{array}$ & Escala de 10 puntos \\
\hline & CuiCiu & $x_{7}$ & $\begin{array}{l}\text { Valoración del cuidado y limpieza } \\
\text { de la ciudad. }\end{array}$ & Escala de 10 puntos \\
\hline \multirow{4}{*}{$\begin{array}{c}\text { Cobertura de } \\
\text { necesidades } \\
\text { vivenciales } \\
\left(\xi_{3}\right)\end{array}$} & Seg & $\mathrm{x}_{8}$ & Valoración de la seguridad percibida. & Escala de 10 puntos \\
\hline & Bell.Pai & $x_{9}$ & Valoración de la belleza natural. & Escala de 10 puntos \\
\hline & PEC & $x_{10}$ & $\begin{array}{l}\text { Valoración del trato recibido del } \\
\text { personal de contacto. }\end{array}$ & Escala de 10 puntos \\
\hline & A Ciud & $x_{11}$ & $\begin{array}{l}\text { Valoración del trato recibido del } \\
\text { ciudadano Tandilense. }\end{array}$ & Escala de 10 puntos \\
\hline $\begin{array}{l}\text { Potencial del } \\
\text { destino }\left(\xi_{4}\right)\end{array}$ & Pot & $\mathrm{x}_{12}$ & $\begin{array}{l}\text { Valoración de lo que "quedó por hacer" } \\
\text { en próximas visitas. }\end{array}$ & Escala de 10 puntos \\
\hline \multirow{3}{*}{$\begin{array}{l}\text { Satisfacción } \\
\qquad\left(n_{1}\right)\end{array}$} & Sat & $\mathrm{y}_{1}$ & Grado de satisfacción. & Escala de 10 puntos \\
\hline & IM & $y_{2}$ & $\begin{array}{l}\text { Grado de coincidencia entre imagen } \\
\text { previa y posterior a la visita. }\end{array}$ & Escala de 10 puntos \\
\hline & $\mathrm{AO}$ & $y_{3}$ & Grado de alcance del objetivo de la visita & Escala de 10 puntos \\
\hline \multirow{2}{*}{$\begin{array}{l}\text { Fidelización } \\
\qquad\left(\eta_{2}\right)\end{array}$} & $\operatorname{Rec}$ & $y_{4}$ & $\begin{array}{l}\text { Grado de acuerdo con la afirmación: } \\
\text { "recomendaría visitar Tandil". }\end{array}$ & Escala de 10 puntos \\
\hline & Rep. Vis & $y_{5}$ & $\begin{array}{l}\text { Grado de acuerdo con la afirmación: } \\
\text { "volvería a visitar Tandil". }\end{array}$ & Escala de 10 puntos \\
\hline
\end{tabular}

Fuente: Elaboración propia

\section{3- CONSIDERACIONES SOBRE LA FORMULACIÓN DEL MODELO}

En general un modelo de ecuaciones estructurales se define a partir de dos submodelos (Foguet, 2000): el modelo de medida y el modelo estructural propiamente dicho. Modelos que algebraicamente se expresan del modo siguiente:

El modelo de medida tiene tantas ecuaciones como variables observables se hayan definido, en este caso 17 ecuaciones. Ecuaciones que se acostumbran agrupar en dos conjuntos, uno para las variables exógenas y otro para las variables endógenas, los cuales en forma matricial se expresan del siguiente modo:

Modelo de medida para las variables exógenas:

$$
x=\Lambda_{x} \xi+\delta
$$


Donde $x$ es el vector que contiene las variables aleatorias observables; $\Lambda_{x}$ una matriz que contiene las saturaciones de las variables observables en las variables latentes independientes; $\xi$ un vector que contiene las variables latentes independientes (exógenas); y el vector $\delta$ contiene las variables aleatorias correspondientes a los errores de medida.

Modelo de medida para las variables endógenas

$$
y=\Lambda_{y} \eta+\varepsilon
$$

Donde $y$ es un vector que contiene las variables aleatorias observables; $\Lambda_{y}$ una matriz que contiene las saturaciones de las variables observables en las variables latentes dependientes; $\eta$ un vector que contiene las variables latentes dependientes (endógenas); y el vector $\varepsilon$ contiene las variables aleatorias correspondientes a los errores de medida.

Modelo estructural

Modelo que en notación matricial, puede expresarse del modo siguiente:

$$
\eta=\beta \eta+\Gamma \xi+\zeta
$$

Donde $\eta$ es el vector que contiene las variables latentes endógenas; $\xi$ el vector de variables latentes exógenas; $\beta$ la matriz des coeficientes de regresión de las variables endógenas sobre las exógenas; $\Gamma$ la matriz de coeficientes de regresión de las variables exógenas sobre las variables endógenas; y $\zeta$ el vector de errores de regresión que resultan de predecir las variables endógenas a partir de las exógenas. Se asume que las variables latentes contenidas en $\eta$ y $\xi$ no están correlacionadas y cualquier relación entre ellas debe estar contenida en los pesos de regresión correspondientes. Además se asume que la matriz $\beta$ no es singular.

\section{4.- SOBRE LOS SUPUESTOS}

En general, para su estimación la mayoría de los modelos matemáticos requieren el cumplimiento de determinados supuestos, en este caso SEM comparte los supuestos de: normalidad multivariante, observaciones independientes, muestra aleatoria de los encuestados y linealidad de todas las relaciones. 


\section{RESULTADOS}

Como primer paso se evaluaron el cumplimiento de los supuestos requeridos por el modelo:

- Supuesto de normalidad: Como se muestra en la Tabla 2 el ratio crítico (c.r) para el coeficiente de kurtosis multivariante es 1,757 inferior a 1,96, implica la aceptación la hipótesis de que las observaciones sigan una distribución normal multivariante al nivel o,05.

Tabla 2. Evaluación de la normalidad de las variables

\begin{tabular}{|c|c|c|c|c|}
\hline Variable & Asimetría & Razón Crítica (c.r) & Kurtosis & Razón Crítica (c.r) \\
\hline Imagen &, 256 & 1,480 &, 291 &, 289 \\
\hline AtenciónCiud &, 226 & 1,595 &, 549 & 1,316 \\
\hline Seg &, 315 & 1,805 &, 225 &, 542 \\
\hline Gastronomía &, 127 &, 546 &, 489 & 1,113 \\
\hline Precios &, 045 &, 081 &, 556 & 1,215 \\
\hline InstalaciónPaseos &, 029 & 1,013 &, 782 & 1,720 \\
\hline Recomendaría &, 111 & 1,222 &, 651 & 1,538 \\
\hline RepVis &, 211 & 1,477 &, 429 &, 591 \\
\hline Pot &, 180 & 1,094 &, 274 &, 883 \\
\hline AlcanceObj &, 244 & 1,131 &, 783 & 1,068 \\
\hline Sat &, 157 &, 651 &, 612 & 1,250 \\
\hline BellezaPaisaje &, 344 & 1,326 &,- 611 & $-1,102$ \\
\hline PEC &, 099 &, 274 & 1,000 & 1,836 \\
\hline ConservacionMA &, 296 & 1,271 &, 011 &, 015 \\
\hline CuiCiu &, 535 & 1,280 &, 188 &, 373 \\
\hline Accesos &, 184 &, 675 &, 503 & 1,217 \\
\hline Alojamiento &, 101 &, 208 &, 729 & 1,043 \\
\hline Multivariante & & & 8,479 & 1,757 \\
\hline
\end{tabular}

Fuente: Elaboración propia con cálculos realizados con AMOS 19.

- Los supuestos de independencia de las observaciones y de aleatoriedad de la muestra de los encuestados están garantizados por el procedimiento utilizado para la recolección de datos que fue descripto en 3.1 .

- Respecto a la linealidad de relaciones, se optó por la matriz de correlaciones ${ }^{2}$, explicitada en la Tabla 3, en la cual queda expresado la linealidad entre cada par de variables. 
Tabla 3: Matriz de correlaciones

\begin{tabular}{|c|c|c|c|c|c|c|c|c|c|c|c|c|c|}
\hline & NA & $A C C$ & SG & BellPai & InsPas & CuiCiu & PEC & ACiud & Pot & $\mathrm{AO}$ & Sat & RepVis & $\operatorname{Rec}$ \\
\hline NA & 1 & & & & & & & & & & & & \\
\hline ACC & ,431(** & 1 & & & & & & & & & & & \\
\hline SG & ,5831** &, $7461^{* *}$ & 1 & & & & & & & & & & \\
\hline BellPai & , $4161^{* *}$ & \begin{tabular}{|l}
, $5911^{*}$ \\
\end{tabular} &, $5061^{* *}$ & 1 & & & & & & & & & \\
\hline InsPas & ,291(** & $4711^{* *}$ & ,405(** &, $5611^{* *}$ & 1 & & & & & & & & \\
\hline CuiCiu & ,253(* &, $2261^{* * *}$ & , 555 (** $^{* *}$ & ,667(** & ,390(* & 1 & & & & & & & \\
\hline PEC & ,7761** &, $5221^{* *}$ & ,412(** & ,371(** &, $3061^{* *}$ &, $3381^{* *}$ & 1 & & & & & & \\
\hline ACiud &, $3551^{* * *}$ & ,277(** &, $3851^{* *}$ &, $2921^{* *}$ &, $4021^{* *}$ & ,318(** & ,805(** & 1 & & & & & \\
\hline Pot & ,255(** & ,230(** & ,345(* & ,183 & ,229(* & ,224(* & ,324(** & ,369/** & 1 & & & & \\
\hline $\mathrm{AO}$ & ,517(** &, $5091^{* *}$ & , $4811^{* * *}$ &, $4611^{*}$ &, $5322^{* *}$ &, $4211^{* *}$ & ,918(* & , 829 (** & ,2931** & 1 & & & \\
\hline Sat & ,497(** & ,393(** &, $523(* *$ & , $4022^{* *}$ &, $3811^{* *}$ & ,285(** & ,831(** & ,744(** & ,388(** & ,842(** & 1 & & \\
\hline RepVis &, $3681^{* * *}$ &, $3161^{* *}$ & ,322(** &, $305\left(^{* *}\right.$ &, $3271^{*}$ & ,246/** & ,771(* & ,692(** & ,797(** & ,602(** &, $7361^{* *}$ & 1 & \\
\hline $\operatorname{Rec}$ &, $5011^{* *}$ & ,417(** &, $5121^{* *}$ & $4333^{* *}$ & $476 * *$ & ,305(** & ,755(** &, $7577^{* *}$ & ,3331** & ,788/** & $8191^{* * *}$ & ,661(** & 1 \\
\hline $\mathrm{IM}$ & ,519(** & ,502(** &, $509\left(^{* *}\right.$ &, $4900^{* *}$ &, $5431^{* *}$ &, $3761^{* *}$ & ,700(** & ,712(** &, 366 (** $^{*}$ &, 800 (** & 897 (** $^{* *}$ &, $6761^{* *}$ & ,888(* \\
\hline
\end{tabular}

** La correlación es significativa al nivel 0,01 (bilateral).

* La correlación es significante al nivel 0,05 (bilateral). Fuente: Elaboración propia

\subsection{ANÁLISIS DEL MODELO}

Según lo explicitado anteriormente, el método de ecuaciones estructurales se ha utilizado para confirmar hipótesis basadas en la teoría que subyace a la elaboración del modelo. A tal efecto, se analizan dos modelos: modelo saturado y modelo no saturado. La estimación para ambos casos se realizó utilizando el método de Máxima Verosimilitud.

Mediante el modelo saturado se confirmaron las relaciones postuladas y se detectó la existencia de relaciones espurias, objetivo de la especificación del modelo en este caso.

La Fig. 3 presenta gráficamente el modelo con las correspondientes estimaciones y los índices de ajuste que se decidió utilizar. 


\begin{tabular}{c}
\hline SILVINA ETCHEVERRÍA - ANA MARÍA LEGATO - PATRICIA LAURA RÁBAGO \\
$>118$ DETERMINANTES DE FIDELIDAD EN EL SERVICIO TURÍSTICO UN MODELO DE RELACIONES CAUSALES \\
Pags. $104-127$ \\
\hline
\end{tabular}

Figura 3. Modelo Saturado.

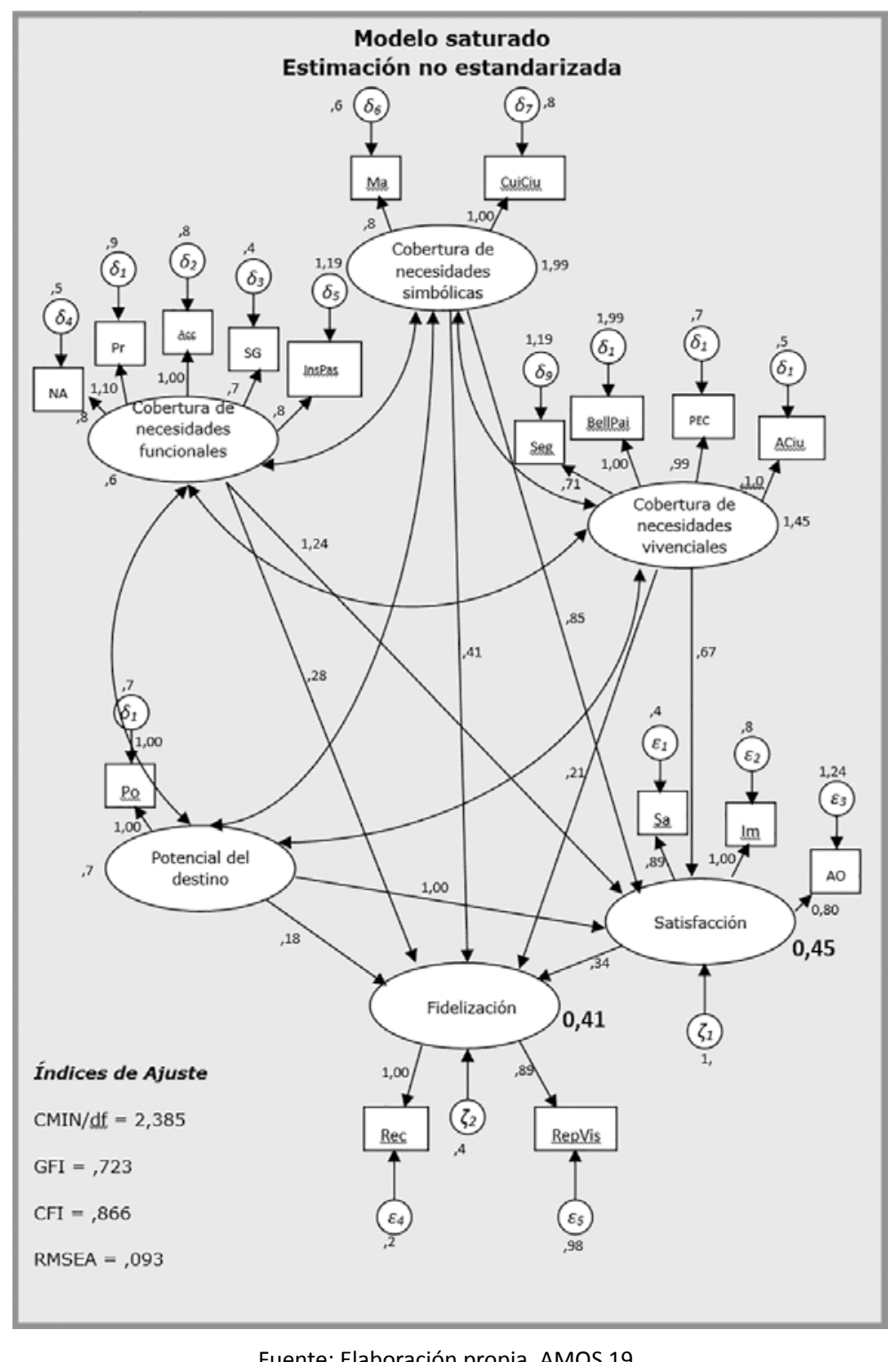

A efectos de la evaluación del modelo, se optó por los índices que se exponen a continuación, generalmente los mas usados en la literatura: $\mathrm{CMIN} / \mathrm{df}\left(\chi^{2}\right.$ relativo o índice de bondad de ajuste) está dentro de los límites del rango considerado aceptable, (Kline, 1998) al ser inferior a 3. Los índices de ajuste comparativo GFI y el CFI asumen valores levemente superiores a los puntos de corte $(0,90)$ (Bentler, 1990) lo cual indica un ajuste bueno. El valor de RMSEA (Root Mean Square Error) es los suficientemente cercano a cero (Kline, 1998) como para considerar un ajuste adecuado. Los valores de los índices conjuntamente con la estimación positi- 
va de las varianzas de los errores (ver Fig. 3), confirman que la solución hallada es admisible.

En la Tabla 4 se presentan las estimaciones para las varianzas del Modelo Saturado. Se puede observar que todas ellas son positivas, lo que implica que la solución hallada es admisible.

Tabla 4. Estimación de varianzas para el Modelo Saturado

\begin{tabular}{|c|c|c|}
\hline Varianzas & Estimación & $\mathrm{p}$-valor \\
\hline Nec. Simb & 1,995 & ${ }^{* * *}$ \\
\hline Nec. Viv & 1,454 & ${ }^{* * *}$ \\
\hline Nec Funcio &, 640 & ${ }^{* * *}$ \\
\hline Potencial &, 751 &, 006 \\
\hline$\zeta 1$ & 1,000 & ${ }^{* * *}$ \\
\hline$\zeta 2$ &, 432 &, 007 \\
\hline$\delta 1$ &, 990 & ${ }^{* * *}$ \\
\hline$\delta 2$ &, 809 & $* * *$ \\
\hline$\delta 3$ &, 399 & .012 \\
\hline$\delta 4$ &, 527 & $* * *$ \\
\hline$\delta 5$ & 1,190 & $* * *$ \\
\hline$\delta 6$ &, 661 & $* * *$ \\
\hline$\delta 7$ &, 834 & $* * *$ \\
\hline$\delta 9$ & 1,193 & $* * *$ \\
\hline$\delta 10$ & 1,991 & $* * *$ \\
\hline$\delta 11$ &, 728 & $* * *$ \\
\hline$\delta 12$ &, 554 & $* * *$ \\
\hline$\delta 13$ &, 786 & $* * *$ \\
\hline$\varepsilon 1$ &, 442 & $* * *$ \\
\hline$\varepsilon 2$ &, 876 & $* * *$ \\
\hline$\varepsilon 3$ & 1,240 & $* * *$ \\
\hline$\varepsilon 4$ &, 209 & $* * *$ \\
\hline$\varepsilon 5$ &, 977 & $* * *$ \\
\hline & & \\
\hline
\end{tabular}

Fuente: Elaboración propia. AMOS 19.

En la Tabla 5 se resumen las estimaciones de los coeficientes estructurales del Modelo Saturado. Se observa que todos los coeficientes estimados son positivos lo que da cuenta de relaciones directas entre cada par de variables.

Se puede apreciar, como era de esperar, que las relaciones presentan distintas intensidades según las variables consideradas. Y, en respuesta al objetivo del planteo de este modelo, se rescata que no todas las estimaciones son significativas, como tal es el es el caso de las relaciones entre Fidelización y Cobertura de necesidades funcionales; Fidelización y Cobertura de necesidades simbólicas y, Fidelización, y Cobertura de necesidades vivenciales; Satisfacción y Potencial del destino, estimaciones que conducen a la modificación del modelo.

Cabe destacar que si bien el modelo cumple el ajuste requerido por la teoría las cuatro relaciones no significativas (Tabla 5) son las que, desde el punto de vista conceptual, parecían tener menos relevancia a priori del estudio de campo y que no presentan un correlato en la hipótesis. Es razonable pensar que las coberturas de necesidades funcionales, simbólicas y 
SILVINA ETCHEVERRÍA - ANA MARÍA LEGATO - PATRICIA LAURA RÁBAGO DETERMINANTES DE FIDELIDAD EN EL SERVICIO TURÍSTICO UN MODELO DE RELACIONES CAUSALES Pags. 104-127

Tabla 5. Estimaciones de los coeficientes estructurales del Modelo Saturado.

\begin{tabular}{|c|c|c|c|c|}
\hline \multicolumn{3}{|c|}{ Coeficiente Estructural } & $\begin{array}{c}\text { Coeficiente } \\
\text { estimado }\end{array}$ & $\begin{array}{c}\text { Nivel de } \\
\text { significación }\end{array}$ \\
\hline Satisfacción & $<---\gamma_{11}$ & Cobertura necesidades funcionales & 1,236 & 005 \\
\hline Satisfacción & $<--\gamma_{12}$ & Cobertura necesidades simbólicas & 845 & $* * *$ \\
\hline Satisfacción & $<---\gamma_{13}$ & Cobertura necesidades vivenciales & 671 & 011 \\
\hline Satisfacción & $<--\rangle_{14}$ & Potencial del destino & 1,000 & ,149 \\
\hline Fidelización & $<--\gamma_{21}$ & Cobertura necesidades funcionales & ,281 & ,411 \\
\hline Fidelización & $<--Y_{22}$ & Cobertura necesidades simbólicas & ,408 & ,199 \\
\hline Fidelización & $<--Y_{23}$ & Cobertura necesidades vivenciales & ,213 & 246 \\
\hline Fidelización & $<--\beta_{21}$ & Satisfacción & ,344 & $* * *$ \\
\hline Fidelización & $<--\gamma_{24}$ & Potencial del destino & 177 & 001 \\
\hline Alojamiento & $<--\lambda_{11}$ & Cobertura necesidades funcionales & 843 & $* * *$ \\
\hline Accesos & $<--\lambda_{21}$ & Cobertura necesidades funcionales & 1,000 & 010 \\
\hline Gastronomia & $<--\lambda_{31}$ & Cobertura necesidades funcionales & ,736 & $* * *$ \\
\hline Precios & $<--\lambda_{41}$ & Cobertura necesidades funcionales & 1,100 & \\
\hline InstalacionPaseos & $<--\lambda_{51}$ & Cobertura necesidades funcionales & 802 & $* * *$ \\
\hline ConservacionMA & $<--\lambda_{61}$ & Cobertura necesidades simbólicas & 888 & $* * *$ \\
\hline CuidadoCiudad & $<--\lambda_{72}$ & Cobertura necesidades simbólicas & 1,000 & \\
\hline Seg & $<--\lambda_{83}$ & Cobertura necesidades vivenciales & ,712 & $* * *$ \\
\hline BellezaPaisaje & $<---\lambda_{73}$ & Cobertura necesidades vivenciales & 1,000 & \\
\hline PEC & $<--\lambda_{103}$ & Cobertura necesidades vivenciales & 993 & $* * *$ \\
\hline AtenciónCiud & $<--\lambda_{113}$ & Cobertura necesidades vivenciales & 1,022 & $* * *$ \\
\hline Sat & $<---\lambda_{11}^{y}$ & Satisfacción & 891 & $* * *$ \\
\hline Imagen & $<--\lambda_{21}^{y}$ & Satisfacción & 1,000 & \\
\hline AlcanceObj & $<--\lambda_{31}^{y}$ & Satisfacción & 804 & $* * *$ \\
\hline Pot & $<--\lambda_{124}^{y}$ & Potencial del destino & 1,000 & \\
\hline Recomendaria & $<--\lambda_{42}^{v}$ & Fidelización & 1,000 & \\
\hline RepVis & $<---\lambda_{52}^{v}$ & Fidelización & 888 & $* * *$ \\
\hline
\end{tabular}

Fuente: Elaboración propia.AMOS 19

vivenciales impacten en la sensación de satisfacción y que, sólo a través de ésta, influyan en la fidelidad del cliente y no directamente en esta última. Por otra parte un destino con mayor o menor potencial (entendido éste como la posibilidad de realizar otras actividades en futuras visitas) debería demostrar una influencia en la fidelidad del cliente y no así en la satisfacción.

Dado entonces que estas relaciones son débiles a la hora de explicar la satisfacción y fidelidad se decide por un nuevo modelo que sólo considere aquellas relaciones que han resultado significativas, se ganaría en parsimonia a la hora de explicar y predecir relaciones.

Bajo las consideraciones anteriores se elaboró un nuevo modelo, Modelo no Saturado (Figura 4) que incluye únicamente las relaciones que han resultado significativas en el modelo anterior. 
Figura 4.Modelo No Saturado.

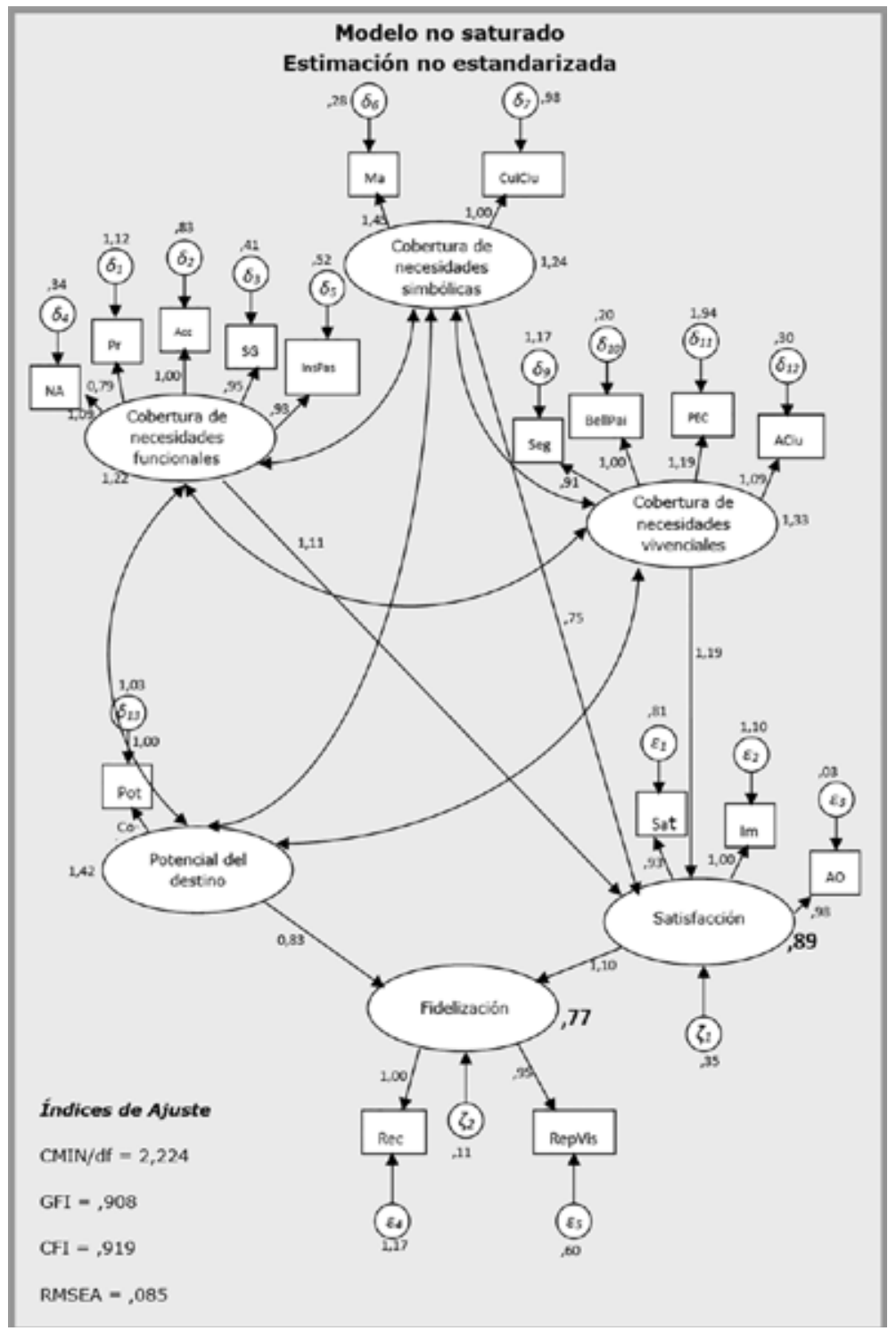

Fuente: Elaboración propia.AMOS 19

En el análisis de los índices de ajuste se aprecia un valor de CMIN/df $(2,224)$ dentro del rango aceptable de valores. En esta oportunidad el GFI $(0,908)$ y el CFI $(0,919)$ superan los valores de corte convencionalmente establecidos, valores que llevan a aceptar la validez del modelo. Por otro lado el valor del RMSEA es ligeramente inferior al del modelo saturado.

Las estimaciones para las varianzas son positivas lo que implica que la solución hallada es admisible (Figura 4).

La Tabla 5 exhibe las estimaciones de los coeficientes estructurales del Modelo no Saturado en la que se puede observar que todas las estimaciones de los parámetros resultan significativas. 
Tabla 6. Estimaciones de los coeficientes estructurales del Modelo No Saturado.

\begin{tabular}{|c|c|c|c|c|}
\hline \multicolumn{3}{|c|}{ Coeficiente Estructural } & $\begin{array}{c}\text { Coeficiente } \\
\text { estimado }\end{array}$ & $\begin{array}{c}\text { Nivel de } \\
\text { significación }\end{array}$ \\
\hline Satisfacción & $<---\gamma_{11}$ & Cobertura necesidades funcionales & 1,109 & 021 \\
\hline Satisfacción & $<---\gamma_{12}$ & Cobertura necesidades simbólicas & 748 & $* * *$ \\
\hline Satisfacción & $<---\gamma_{13}$ & Cobertura necesidades vivenciales & 1,192 & ,009 \\
\hline Fidelización & $<--\beta_{21}$ & Satisfacción & 1,102 & $* * *$ \\
\hline Fidelización & $<--\gamma_{24}$ & Potencial del destino & ,833 & 002 \\
\hline Alojamiento & $<--\lambda_{11}$ & Cobertura necesidades funcionales & 1,088 & $* * *$ \\
\hline Accesos & $<--\lambda_{21}$ & Cobertura necesidades funcionales & 1,000 & $* * *$ \\
\hline Gastronomia & $<--\lambda_{31}$ & Cobertura necesidades funcionales & 951 & $* * *$ \\
\hline Precios & $<--\lambda_{41}$ & Cobertura necesidades funcionales & ,794 & \\
\hline InstalacionPaseos & $<--\lambda_{51}$ & Cobertura necesidades funcionales & ,932 & $* * *$ \\
\hline ConservacionMA & $<--\lambda_{61}$ & Cobertura necesidades simbólicas & 1,451 & $* * *$ \\
\hline CuidadoCiudad & $<--\lambda_{72}$ & Cobertura necesidades simbólicas & 1,000 & \\
\hline Seg & $<--\lambda_{83}$ & Cobertura necesidades vivenciales & 908 & $* * *$ \\
\hline BellezaPaisaje & $<--\lambda_{73}$ & Cobertura necesidades vivenciales & 1,000 & \\
\hline PEC & $<--\lambda_{103}$ & Cobertura necesidades vivenciales & 1,185 & $* * *$ \\
\hline AtenciónCiud & $<--\lambda_{113}$ & Cobertura necesidades vivenciales & 1,085 & $* * *$ \\
\hline Sat & $<--\lambda_{11}^{y}$ & Satisfacción & 927 & $* * *$ \\
\hline Imagen & $<---\lambda_{21}^{y}$ & Satisfacción & 1,000 & \\
\hline AlcanceObj & $<---\lambda_{31}^{y}$ & Satisfacción & ,983 & $* * *$ \\
\hline Pot & $<--\lambda_{124}^{y}$ & Potencial del destino & 1,000 & \\
\hline Recomendaría & $<---\lambda_{42}^{y}$ & Fidelización & 1,000 & \\
\hline RepVis & $<---\lambda_{52}^{y}$ & Fidelización & ,945 & $* * *$ \\
\hline
\end{tabular}

Fuente: Elaboración propia.AMOS 19

El modelo estructural, utilizando la ecuación (3) queda expresado simbólicamente como sigue 3 :

$\eta_{2}=\beta_{21} \xi_{1}+\lambda_{24} \xi_{4}$

$$
\eta_{1}=\lambda_{11} \xi_{1}+\lambda_{12} \xi_{2}+\lambda_{13} \xi_{3}
$$

en tanto que, reemplazado a los símbolos por su correspondiente variable, resulta

Satisfacción $=1.11$ Cobertura Necesidades Funcionales + 0.75 Cobertura Necesidades Simbólicas + 1.192 Cobertura Necesidades Vivenciales

Fidelización $=1.10$ Satisfacción +0.83 Potencial de destino

Ecuaciones que indican que las relaciones consideradas en el modelo y postuladas en las Hipótesis H1a, H1b, H1c, H2a y H2b son confirmadas. Se aprecia que el 89\% de la variabilidad en la Satisfacción manifestada por los turistas está explicada por las variaciones en la Cober- 
tura de necesidades funcionales, vivenciales y simbólicas. Mientras que el 77\% de la variabilidad de la Fidelización puede explicarse por la variabilidad en la Satisfacción percibida y del Potencial del destino.

En resumen, los resultados obtenidos permiten afirmar con una confianza del 95\% que, en efecto, dar cobertura a las necesidades vivenciales, simbólicas y funcionales influye positivamente en la satisfacción del turista, lo cual favorece, junto al potencial del destino, la fidelidad del visitante al destino.

Atendiendo a los valores de los coeficientes estructurales expuestos en la Tabla 6 y en la Figura 4 se puede apreciar que la Cobertura de necesidades Vivenciales es la que más impacta en la Satisfacción presentando un coeficiente estructural $\gamma_{13}=1,192$, en tanto que el indicador de mayor peso es la Atención recibida por parte del Personal de Contacto $\left(\lambda_{103}=1.19\right)$ seguido de la Atención recibida por parte del ciudadano de Tandil $\left(\lambda_{113}=1.09\right)$.

Por otro lado, la relación entre la Cobertura de necesidades funcionales y la Satisfacción es de considerable magnitud $\left(\gamma_{11}=1,11\right)$ siendo el indicador de mayor peso el de Nivel de Alojamiento $\left(\lambda_{11}=1.09\right)$ y el de menor relevancia el Nivel de Precios $\left(\lambda_{41}=0.79\right)$. De igual modo se puede observar una interesante relación entre la Satisfacción y la Cobertura de necesidades simbólicas $\left(\gamma_{12}=0,748\right)$, aunque con pesos de menor magnitud, pero confirman la relación entre los constructos mencionados.

Asimismo, la Fidelización del turista está determinada en gran parte por la satisfacción alcanzada durante su estadía $\left(\beta_{21}=1,10\right)$ y también, aunque en menor medida, por el Potencial del destino Tandil $\left(\gamma_{24}=0,83\right)$.

\section{DISCUSIÓN}

Luego del estudio de los datos relevados, y entendiendo que se trata de un análisis confirmatorio del modelo, existen indicios suficientes para indicar que el modelo explica las hipótesis enunciadas. Los elevados coeficientes estructurales entre las variables estimadas en el análisis del Modelo muestran que, efectivamente, la actuación sobre el conjunto de Cobertura de necesidades tiene una influencia relevante sobre la Satisfacción, llegando a explicar el 89\% de su variabilidad confirmándose de esta manera las hipótesis H1a, H1b, y H1c. Sólo el 11\% de la varianza no explicada puede deberse a factores externos no contemplados en el modelo, por tal motivo no son objeto de estudio en el presente trabajo.

Este resultado invita a prestar especial atención a cada una de las categorías en que se han agrupado las necesidades de los turistas, y a desagregar cada una de ellas analizando los indicadores que las determinan. 
En primer lugar se evalúa el impacto de la Cobertura de necesidades vivenciales sobre la Satisfacción, siendo el indicador con mayor peso la atención recibida por parte del personal de contacto, y en menor escala la atención recibida por ciudadano de Tandil.

En segundo lugar se puede establecer que la Satisfacción depende también del nivel de Cobertura de necesidades funcionales apreciado por el turista $\left(\gamma_{11}=1,109\right)$. Se debe destacar que el indicador que mayor influencia marca sobre la Cobertura de necesidades funcionales es el Nivel de Alojamiento $\left(\lambda_{11}=1,09\right)$, en tanto que el Nivel de precios presenta menor relevancia $\left(\lambda_{41}=0,79\right)$. Es decir que los visitantes pudieron manifestar su estado de satisfacción aun cuando consideraban que el nivel de precios era alto. Así, por ejemplo, una evaluación positiva del alojamiento recibido se ve reflejada en la Satisfacción casi con independencia del precio pagado.

En tercer lugar el trabajo de campo confirma la relación entre la Cobertura de necesidades simbólicas con la Satisfacción del turista $\left(\gamma_{12}=0,748\right)$. La percepción del medio ambiente cuidado como así también la imagen de la ciudad prolija y pulcra complacen al visitante.

Finalmente se puede precisar que a partir del modelo se confirma la relación positiva entre la Satisfacción y el Potencial del destino con la Fidelidad. La variabilidad de ésta es explicada en un $77 \%$ por aquéllas. La lealtad del turista, entendida como propósito de repetir la visita o recomendar el destino, está determinada en gran parte por la Satisfacción alcanzada durante su estadía y también, aunque en menor medida, por el Potencial del destino Tandil. En otras palabras, un turista volverá a visitar la ciudad o la recomendará fundamentalmente si se va satisfecho, pero también influirá en esa decisión la sensación de que el destino puede brindarle la posibilidad de vivir nuevas experiencias.

\section{CONCLUSIÓN}

El estudio realizado en la presente investigación explica, a través de un modelo conceptual, los factores que determinan la satisfacción y fidelidad de los turistas que visitan un destino turístico en particular.

La contextualización del hecho turístico a través del concepto de destino turístico y producto integral turístico permiten sentar las bases para identificar rasgos genéricos de la demanda y particularmente los factores que determinan su satisfacción.

A la luz de los resultados que arroja el modelo diseñado y estadísticamente confirmado empleando Modelos de Ecuaciones Estructurales se ha podido arribar a diversas conclusiones.

Se pudo establecer como primer factor determinante de la satisfacción del turista el nivel de cobertura de necesidades vivenciales, encontrando que el trato recibido tanto del personal de contacto como del ciudadano común son los aspectos a los que se les dan mayor importancia.

El factor que le sigue en importancia es el que refleja la cobertura de necesidades funcionales. La percepción de la calidad del alojamiento influye positivamente en la satisfacción 
del turista, con independencia del nivel de precios.

En tercer lugar aparece la cobertura de necesidades vivenciales como influencia positiva sobre la satisfacción, entendiendo que dentro de estas se posiciona principalmente el cuidado medioambiental.

Por último se pudo establecer que la fidelización del turista es consecuencia, por un lado, de la satisfacción con todos los condicionantes examinados precedentemente, y por otro del potencial del destino.

La formulación de un modelo matemático adquiere mayor valor si el mismo puede ser validado en más de un escenario. En este sentido se entiende que, adecuando la definición de los constructos e indicadores correspondientes a otros destinos y/u organizaciones, el modelo propuesto permitirá estudiar la satisfacción y fidelidad de turistas en otros destinos.

\section{REFERENCIAS BIBLIOGRAFICAS}

Bentler, P.M. (1990). Comparative fit indexes in structural models. Psychological Buletin, 107, 238-246.

Bitner, M. J., Booms, B.H. \& Mohr, L.A. (1990). The Service Encounter: Diagnosing favourable and unfavourable incidents. Journal of Marketing, 54 (1), 71-84.

Bowen,D. \& Clarke, L. ( 2002). Reflections on tourists satisfaction research: Past, present and future. Journal on Vacation Marketing 8 (4), 297-308.

Devesa Fernández, M. \& Palacios Picos, A. (2005). Predicciones en el nivel de satisfacción percibida por los turistas a partir de variables motivacionales y de valoración de la visita. Tribuna de economía. ICE. (2) 241-255.

Foguet, Joan Manuel Bautista, (2000). Modelos de Ecuaciones Estructurales. Ed. La Muralla. Madrid.

Goldberger A.S, Duncan O.D, (1973). Structural Equation Models in the Social Sciences. New York Seminar Press.

Gundersen, M.G. \& Olsson, U.H. (1996). Hotel guest satisfaction among business travellers: What are the important factors? The Cornell Hotel and Restaurant Administration Quarterly, 37 (2), 72-81. 


\begin{tabular}{c}
\hline SILVINA ETCHEVERRÍA - ANA MARÍA LEGATO - PATRICIA LAURA RÁBAGO \\
$>126$ DETERMINANTES DE FIDELIDAD EN EL SERVICIO TURÍSTICO UN MODELO DE RELACIONES CAUSALES \\
Pags. $104-127$
\end{tabular}

Jöreskog, K. G., \& Sörbom, D. (1979). Advances in factor analysis and structural equation models. New York: University Press of America.

Kline, R. B. (1998). Principles and practice of structural equation modeling. NewYork: The Guilford Press.

Kotler, Philip, Donald H. Haider e Irving Rein, (1993). Marketing Places. New York: The Free Press.

Kotler, P., Bowen, J. y Markens, J., (1997). Mercadotecnia para Hotelería y Turismo, Prentice Hall, México.

Kozak, M. \& Rimminghton, M. (2000). Tourist Satisfaction with Mallorca, Spain, as an off-Season Holiday Destination. Journal of Travel Research, 38, 260-269.

Nowak, L. y Washburn, J. H. (1998). Antecedents to Client Satisfaction in Business Service, The Journal of Services Marketing, 12 (6), 441-452.

Oliver, R.L. (1980). A Cognitive Model of the Antecedents and Consequences of Satisfaction Decisions. Journal of Marketing Research, 17(4), November. 460-469.

Oyarzún Mendez, E \& Szmulewicz Pablo (2002). ¿Qué, cómo y a quién vender Turismo? Gestión Turística. 4 (1), 27-49.

Rico, R. R., (1993). Total customer satisfaction. Satisfacción y deleite total de los clientes. Buenos Aires, Argentina. Ediciones Macchi.

Spreng, R. A., Mackenzie, S. B., \& Olshavsky, R. (1996). A Reexamination of the Determinants of Consumer Satisfaction. Journal of marketing, 60, 11-32.

Szymanski, D.M. \& Henard, D.H. (2001). Customer Satisfactions: A Meta-Analysis of the Empirical Evidence, Journal of the Academy of Marketing Science, 29, (1), 16-35.

Wirtz, J., Mattila, A.S. \& Tan, R.L.P. (2000). The moderating role of target-arousal on the impact of affect on satisfaction. An examination in the context of service experiences. Journal of Retailing, 76(3), 347-365.

Zins, A.H. (2002). Consumption emotions experience quality and satisfaction: A structural analysis for complainers versus no complainers, Journal of Travel and Tourism 


\section{CURRICULUM VITAE}

\section{Silvina Etchevarria}

Magister en Administración de Negocios. (UNICEN, Argentina). Jefe de trabajos Prácticos de la Facultad de Ciencias Económicas - UNICEN.

silvinaetcheverria@yahoo.com

\section{Ana Maria Legato}

Magister en Administración de Negocios (UNICEN, Argentina - California State University, Los Angeles, EEUU). Profesor Titular Dedicación Exclusiva, Facultad de Ciencias Económicas UNICEN. Profesor Invitado para cursos de Doctorado en la Facultad de Económicas y Empresariales, Universidad de Castilla La Mancha - España, sobre Técnicas Avanzadas de Investigación: Análisis de Datos Multivariados. Profesor del Doctorado en Ciencias de la Gestión, Facultad de Ciencias Económicas - UNLP, sobre Métodos Cuantitativos en Investigación. Profesor responsable de cursos en la Maestría en Dirección de Empresas - UNLP, sobre Estadística y Probabilidades en los Negocios y Pronósticos en los Negocios. Autor de Textos y publicaciones sobre la especialidad. Estudios de Posgrado en el Exterior (Universtà di Siena, Italia). Investigador del CEA (Centro de Estudios en Administración), Facultad de Ciencias Económicas - UNICEN. legato@econ.unicen.edu.ar

\section{Patricia Laura Rábago}

Magister en Comercialización (UNICEN - Argentina, - UFRGS - Universidade Federal do Rio Grande do Sul, Brasil). Master en Desarrollo Económico en América Latina (Universidad Internacional de Andalucía, España). Investigadora del Centro de Estudios en Administración (CEA), Facultad de Ciencias Eco-nómicas, UNICEN. Profesor Adjunto concursado, Facultad Ciencias Económicas - UNICEN. Autor de numerosas publicaciones de la especialidad. rabago@econ.unicen.edu.ar 